\title{
AVALIAÇÃO RADIOGRÁFICA DO PREENCHIMENTO DE CANAIS LATERAIS ARTIFICIAIS UTILIZANDO TRÊS TÉCNICAS DE OBTURAÇÃO
}

\section{RADIOGRAPHIC EVALUATION OF FILLING OF ARTIFICIAL LATERAL CANALS BY USING THREE TECHNIQUES OF FILLING}

\author{
Karoline da Silva Rebouças* \\ Matheus Melo Pithon"* \\ Manoel Matos Neto***
}

\section{RESUMO}

Objetivo: Avaliar radiograficamente a capacidade de obturação dos canais laterais artificiais utilizando-se as técnicas de condensação lateral, híbrida de Tagger e condensação vertical de ondas contínuas (Schilder). Materiais e métodos: Foram utilizados 30 dentes humanos oriundos do Banco de Dentes Humanos da Universidade Estadual do Sudoeste da Bahia, sendo estes divididos em três grupos $(n=10)$ para comparações das técnicas obturadoras. Após o preparo químico-mecânico, foram confeccionados canais laterais artificiais nos dentes, em nível apical, médio e cervical, por intermédio de uma lima \#10 tipo K calibrada em sua ponta a um diâmetro equivalente a uma lima \#20 tipo K. Os dentes foram obturados e em seguida, avaliados com radiografia digital, considerando-se a quantidade de canais simulados que foram preenchidos pelo material obturador. Os resultados foram submetidos à análise estatística com utilização do teste não paramétrico do Qui-quadrado, no qual foi considerada diferença estatística quando $\mathrm{p}<0.05$. Resultados: A técnica de condensação lateral apresentou diferença estatisticamente significante $(p<0.05)$ quando comparada à híbrida de Tagger e condensação vertical de ondas contínuas. Porém, não se evidenciou diferença estatisticamente significante entre as técnicas híbrida de Tagger e condensação vertical de ondas contínuas ( $p>0.05)$. Conclusão: Nenhuma das técnicas obturadoras avaliadas foi capaz de preencher todos os canais laterais simulados. Houve diferença significativa quando se comparou a técnica de condensação lateral com as outras técnicas avaliadas. Entre as técnicas híbrida de Tagger e condensação vertical de ondas contínuas não ocorreu diferença estatística.

DESCRITORES: Endodontia • Obturação de canal radicular • Radiografia dentária.

\section{ABSTRACT}

Objective: The aim of this study is to evaluate radiographically the ability of filling of lateral canals by using the lateral condensation technique, Tagger's hybrid and vertical condensation technique for continuous waves (Schilder). Materials and methods: 30 human teeth were used from the Human Teeth Bank of the State University of Southwestern Bahia. They were divided into three groups $(n=10)$ in onder to compare the filling techniques. After chemical-mechanical preparation, artificial lateral canals were made in teeth, at apical, middle and cervical level through K-type file \#10 calibrated at its tip to a equivalent to the K-type file \#20. The teeth were filled and then evaluated with digital radiography considering the amount of simulated canals wich were filled by filling material. The results were analyzed statistically using the nonparametric chi-square. In this case, it was considered a statistical difference at $\mathrm{p}<0.05$. Results: The lateral condensation technique showed a statistically significant difference $(p<0.05)$ when compared to Tagger's hybrid and vertical condensation technique for continuous wave (Schilder). But it was not realized statistically significant difference between the techniques Tagger's hybrid and vertical condensation technique for continuous waves (schilder) $(p>0.05)$. Conclusion: None of filling techniques evaluated was able to fill all the simulated lateral canals. There were significant differences when compared to lateral condensation technique with other techniques evaluated. Among the techniques Tagger's

DESCRIPTORS: Endodontics • Root canal fillings • Dental radiography.

\footnotetext{
* Acadêmica do Curso de Odontologia da Universidade Estadual do Sudoeste da Bahia.

** Professor Doutor do Curso de Odontologia da Universidade Estadual do Sudoeste da Bahia

*** Professor Mestre do Curso de Odontologia da Universidade Estadual do Sudoeste da Bahia
} 


\section{N T R O D UÇÃ O}

A obturação do sistema de canais radiculares significa preenchê-los com um material inerte e antisséptico, tendo como objetivo primordial promover um selamento hermético e tridimensional permanente ${ }^{1-3}$.

Na terapia endodôntica, a fase de obturação merece um destaque especial, pois esta objetiva manter as condições biológicas obtidas nas fases anteriores do tratamento, determinando, então, o coroamento do tratamento endodôntico ${ }^{4,5}$. Destarte, a preocupação dos pesquisadores tem sido cada vez maior no sentido de encontrar materiais e técnicas ideais visando melhorar a qualidade da terapia endodôntica, bem como facilitar a sua execução ${ }^{6}$.

Com os avanços no tratamento endodôntico, nota-se um aumento no interesse pelo estudo da anatomia interna dentária $^{2,7}$. Isso ocorre devido à necessidade de reconhecer as variações existentes no sistema de canais radiculares, já que o sucesso do tratamento endodôntico depende da localização, limpeza, desinfecção e obturação deles. Os canais laterais, acessórios e deltas apicais, quando não preenchidos, podem alimentar uma infecção, mesmo estando o canal principal completamente obturado ${ }^{8}$. A existência dessas áreas permite intuir que elas poderão abrigar restos orgânicos contaminados e, consequentemente, viabilizar um fracasso endodôntico ${ }^{9}$. Assim, observa-se a necessidade da efetividade de uma obturação tridimensional, preenchendo todo sistema de canais.

$\mathrm{Na}$ endodontia, a radiografia colabora na constatação final do selamento adequado dos condutos, bem como é de grande importância na avaliação periódica pós-tratamento realizado e na verificação da integridade do periápice ${ }^{10}$.

No que diz respeito às técnicas empregadas, todas têm um objetivo comum: reunir qualidade com praticidade ${ }^{11}$. $\mathrm{Na}$ busca da melhoria na qualidade do selamento, diversas técnicas de obturação foram desenvolvidas nas últimas décadas $^{7,12}$. Cada autor atribui à sua técnica superioridade em relação às demais ${ }^{13,14}$. No entanto, novas pesquisas são neces- sárias para comprovar efetivamente qual seria a melhor técnica. Baseando-se nessa premissa, a proposta do presente estudo é avaliar radiograficamente a capacidade de obturação dos canais laterais artificiais utilizando as técnicas de condensação lateral, híbrida de Tagger e condensação vertical de ondas contínuas.

\section{MATERIAS E MÉTODOS}

O estudo foi realizado no laboratório pré-clínico do módulo de Odontologia da Universidade Estadual do Sudoeste da Bahia (UESB). Trata-se de um estudo transversal quantitativo. Consiste numa pesquisa do tipo laboratorial, um estudo in vitro. $\mathrm{O}$ trabalho apresenta aprovação no Comitê de Ética em Pesquisa da Universidade Estadual do Sudoeste da Bahia sob protocolo de $n^{\circ}$. 127/2011.

\section{Seleção e preparo dos dentes}

Foram utilizados 30 dentes unirradiculares oriundos do Banco de Dentes Humanos $(\mathrm{BDH})$ da Universidade Estadual do Sudoeste da Bahia. O critério de seleção consistiu em unidades dentais hígidas, com canal único, longo e volumoso, para melhor confecção e visualização das obturações dos canais laterais artificiais. Estes compreenderam caninos e incisivos centrais.

As unidades dentárias, depois de selecionadas, foram limpas e conservadas em formol a $10 \%$ até o momento do uso, sendo lavadas em água corrente por uma hora e secas com gaze. Em seguida, o acesso coronário foi realizado com pontas diamantadas esféricas de diâmetro compatível com o volume coronário, em alta rotação com refrigeração ar/água, sendo depois substituídas pela fresa tronco-cônica de ponta inativa Endo-Z (Denstsply Maillefer, Ballaigues, Suíça), para realização do desgaste compensatório, bem como acabamento das paredes circundantes.

Após o acesso, realizou-se o preparo químico-mecânico dos canais radiculares, por meio da técnica MRA ${ }^{15}$. Essa técnica se dá pela sequência: cateterismo (localização dos canais); ampliação reversa ou step-down (pré-alargamento com limas \#15 á \#40 tipo K (Maillefer, Ballaigeus, Suíça); uso de brocas de Gattes (Densts-
REBOUÇAS KS

PITHON MM

MATOS NETO M

AVALIAÇÃO

RADIOGRÁFICA DO

PREENCHIMENTO DE

CANAIS LATERAIS

ARTIFICIAIS

UTILIZANDO TRÊS

TÉCNICAS DE

obtuRação
REV, ODONTOL.

UNIV. CID. SÃO

PAULO

$2013 ; 25(2): 126$ -

34, MAIO-AGO 
REBOUÇAS KS

PITHON MM

MATOS NETO M

AVALIAÇÃO RADIOGRÁFICA DO PREENCHIMENTO DE CANAIS LATERAIS ARTIFICIAIS UTILIZANDO TRÊS TÉCNICAS DE OBTURAÇÃO

\section{8}

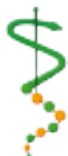

REV, ODONTOL.

UNIV, CID, SÃO

PAULO

2013; $25(2): 126$

34, MAIO-AGO ply Maillefer, Brasil) \#4-1(alargamento dos terços cervical e médio); odontometria (mensuração do término apical com utilização de uma lupa recuando $1 \mathrm{~mm}$ da saída da lima pelo forame apical); realização da patência com limas \#10, \#15 e \#20 tipo K; preparo apical (criação do batente apical até lima memória); recuo progressivo (step-back); recapitulação constante do comprimento de patência (lima \#20 tipo $\mathrm{K})$.

Para o preparo apical foram utilizadas limas endodônticas de aço-inoxidável do tipo Flexofile (Maillefer, Ballaigeus, Suíça) até a lima \#45 (lima memória), $1 \mathrm{~mm}$ aquém do comprimento de patência. A lima \#20 tipo K teve como utilidade a manutenção da patência foraminal e assim não permitiu o acúmulo de smear layer no forame apical. O hipoclorito de sódio a 2,5\% (água sanitária marca $Q-B O A \circledast$, Indústria Anhembi S/A, São Paulo, Brasil) foi eleito substância irrigadora de escolha durante todo o processo de irrigação/aspiração da instrumentação.

\section{Confecção dos canais laterais}

Após o preparo químico-mecânico, foram confeccionados canais laterais artificiais nos dentes utilizando uma lima \#10 tipo $\mathrm{K}$ calibrada em sua ponta a um diâmetro equivalente a uma lima \#20 tipo $\mathrm{K}$, por meio de uma régua calibradora endodôntica (Maillefer, Ballaigeus, Suíça). Esses canais são feitos após a instrumentação para evitar interferências no orifício do canal lateral junto ao canal principal, reduzindo o risco de bloqueio dos canais, que seria promovido por raspas de dentina removidas durante o alargamento do canal. Por conseguinte, a lima calibrada é adaptada a um micromotor. Os canais laterais foram criados em níveis apical, médio e cervical a uma distância de 3, 6 e 9 milímetros do ápice respectivamente, sendo dois canais confeccionados em cada altura (um na face mesial e outro na distal), totalizando 6 canais por dente. Em seguida, foram utilizados $3 \mathrm{ml}$ de EDTA (Biodinâmica Química e Farmacêutica Ltda.) para remoção de smear layer, ativando por 30 segundos com Lentulo (Denstsply Maillefer, Suiça) e como solução irrigadora final soro fisiológico.

\section{Obturação}

Em seguida, as 30 raízes foram então divididas aleatoriamente, em três grupos $(n=10)$, sendo obturadas com as seguintes técnicas: condensação lateral (Grupo I), híbrida de Tagger (Grupo II) e condensação vertical de ondas contínuas (Grupo III). É válido ressaltar que todos os dentes foram instrumentados e obturados pelo mesmo operador.

A técnica de condensação lateral (Grupo I) é dada pela sequência clínica: a) seleção do cone principal de guta-percha não calibrado, tamanho M (Endo-Points, Indústria e Comércio Ltda., Rio de Janeiro, Brasil), calibrando-o de acordo com a lima memória utilizada durante a instrumentação; b) desinfecção dos cones de guta-percha mergulhados em hipoclorito de sódio 2,5\% por 10 minutos; c) testar o cone principal com o canal radicular ainda umedecido com soro fisiológico; d) comprovação radiográfica do cone principal - terminando no comprimento de trabalho (CT) $1 \mathrm{~mm}$ aquém do ápice; e) secagem do conduto com cones de papel absorvente \#40 (Dentsply, Indústria e Comércio Ltda., Rio de Janeiro, Brasil); f) preparo do cimento obturador Sealer 26 (Dentsply, Indústria e Comércio Ltda., Rio de Janeiro, Brasil); g) colocação do cimento por meio do próprio cone principal levando-o até o CT; h) condensação lateral ativa com espaçador digital tamanho B-vermelho (Denstsply Maillefer, Suiça) e colocação de cones de guta-percha auxiliares não calibrados, tamanho MF (Endo-Points, Indústria e Comércio Ltda., Rio de Janeiro, Brasil) envolto por cimento até preencher todo o conduto; i) cortar, com a ponta do condensador de Schilder (ODOUS, Belo Horizonte, Minas Gerais, Brasil) aquecida por lamparina, o excesso de material obturador até o limite cervical radicular; j) condensação vertical do material obturador com condensador de Schilder.

Na técnica híbrida de Tagger (Grupo II), foi realizada uma condensação lateral como descrito no Grupo I, porém utilizando-se apenas de 3 a 5 cones de guta-percha acessório não calibrado, tamanho MF. Em seguida, broca Mcspaden \#55 (Dentsply - Maillefer - Suiça) foi utilizada 
em baixa rotação até $02 \mathrm{~mm}$ aquém do comprimento de trabalho, a qual plastificou por atrito toda a guta-percha. O excesso desse material foi removido com a ponta do calcador de Paiva aquecida com lamparina até o limite cervical radicular, sendo depois realizada a condensação vertical com condensador de Schilder.

$\mathrm{Na}$ técnica de condensação vertical de ondas contínuas (Grupo III), utilizou-se a seguinte sequência: escolha do cone de guta-percha principal não calibrado, tamanho $M$ compatível com o tip da lima memória; colocação desse cone juntamente com o cimento obturador no conduto até o comprimento de trabalho; corte do cone na altura do terço cervical com um condutor de calor de ponta FM (Easy Endo - Brasil); utilização do condutor de calor com um stop posicionado a $4 \mathrm{~mm}$ aquém do comprimento de trabaIho visando remover a guta-percha até esse comprimento (fase de DownPack); repreenchimento do espaço vazio com a utilização de brocas de McSpadden \#45 e guta-percha aquecida (fase de Repack); condensação vertical com condensador de Schilder.

\section{Análise e Processamento de Dados}

A efetividade das obturações desses canais foi avaliada radiograficamente e classificada considerando-se a quantidade de canais simulados que foram preenchidos com o material obturador nas diferentes alturas. Essas imagens foram avaliadas, sendo contabilizado o preenchimento dos canais laterais confeccionando-se tabelas para cada grupo por meio de 03 avaliadores previamente calibrados.

Os resultados foram, então, submetidos à análise estatística com utilização do teste não paramétrico do Qui-quadrado, no qual foi considerada a diferença estatística quando $\mathrm{p}<0.05$.

\section{RESULTADOS}

Os resultados demonstraram que a técnica de condensação lateral apresentou a maioria dos canais laterais sem obturar, e naqueles preenchidos constatou-se apenas a presença do cimento, com ênfase para nenhuma obturação em $3 \mathrm{~mm}$ à direita, segundo Tabela 1 abaixo.

Conforme a Tabela 2, foi possível verificar um melhor desempenho da técnica híbrida de Tagger quando comparada à condensação lateral, com preenchimento da maior parte dos canais seja com cimento e/ou guta-percha termoplastificada. Estes apresentaram maior efetividade a nível cervical.

A técnica de condensação vertical de ondas contínuas apresentou a maior frequência de obturação entre as três técnicas comparadas (Tabela 3).

Com essas tabelas os dados foram submetidos à avaliação estatística pelo teste não paramétrico $x^{2}$ (qui-quadrado). Após a análise, comparando-se os grupos e dentro do mesmo grupo, verificou-se diferença estatisticamente significante $(p<0.05)$ na comparação entre 3 e $6 \mathrm{~mm}$ e 6 e $9 \mathrm{~mm}$ da técnica de condensação vertical de ondas contínuas. Nas demais comparações,

Tabela 1. Técnica condensação lateral (Grupo I) - preenchimento de canais laterais

\begin{tabular}{ccccccc}
\hline \hline Dentes & 9mm & $9 \mathrm{~mm}$ & $6 \mathrm{~mm}$ & $6 \mathrm{~mm}$ & $3 \mathrm{~mm}$ & $3 \mathrm{~mm}$ \\
& Esquerda & Direita & Esquerda & Direita & $\begin{array}{c}\text { Esquerda } \\
\text { Direita }\end{array}$ \\
\hline 1 & não & $\operatorname{sim}$ & não & $\operatorname{sim}$ & $\operatorname{sim}$ & não \\
2 & $\operatorname{sim}$ & $\operatorname{sim}$ & $\operatorname{sim}$ & $\operatorname{sim}$ & $\operatorname{sim}$ & não \\
3 & não & não & não & não & $\operatorname{sim}$ & não \\
4 & sim & $\operatorname{sim}$ & não & não & não & não \\
5 & não & não & não & não & não & não \\
6 & não & sim & não & sim & não & não \\
7 & não & não & sim & não & não & não \\
8 & sim & não & sim & não & não & não \\
9 & não & não & não & não & não & não \\
10 & sim & sim & sim & sim & não & não \\
\hline \hline
\end{tabular}

REBOUÇAS KS

PITHON MM

MATOS NETO M

AVALIAÇÃO

RADIOGRÁFICA DO

PREENCHIMENTO DE

CANAIS LATERAIS

ARTIFICIAIS

UTILIZANDO TRÊS

TÉCNICAS DE

OBTURAÇÃO 
REBOUÇAS KS PITHON MM : MATOS NETO M

AVALIAÇÃO RADIOGRÁFICA DO PREENCHIMENTO DE CANAIS LATERAIS ARTIFICIAIS UTILIZANDO TRÊS TÉCNICAS DE OBTURAÇÃO

REV, ODONTOL, UNIV. CID, SÃO PAULO: 2013; 25(2): 126-

34, MAIO-AGO:
Tabela 2. Técnica híbrida de Tagger (Grupo II)-preenchimento de canais laterais

\begin{tabular}{ccccccc}
\hline \hline Dentes & $9 \mathrm{~mm}$ & $9 \mathrm{~mm}$ & $6 \mathrm{~mm}$ & $6 \mathrm{~mm}$ & $3 \mathrm{~mm}$ & $3 \mathrm{~mm}$ \\
\hline 1 & Esquerda & Direita & Esquerda & Direita & Esquerda & Direita \\
2 & $\operatorname{sim}$ & $\operatorname{sim}$ & $\operatorname{sim}$ & $\operatorname{sim}$ & não & $\operatorname{sim}$ \\
3 & $\operatorname{sim}$ & $\operatorname{sim}$ & $\operatorname{sim}$ & $\operatorname{sim}$ & $\operatorname{sim}$ & $\operatorname{sim}$ \\
4 & $\operatorname{sim}$ & $\operatorname{sim}$ & não & $\operatorname{sim}$ & $\operatorname{sim}$ & $\operatorname{sim}$ \\
5 & $\operatorname{sim}$ & $\operatorname{sim}$ & $\operatorname{sim}$ & $\operatorname{sim}$ & $\operatorname{sim}$ & $\operatorname{sim}$ \\
6 & $\operatorname{sim}$ & $\operatorname{sim}$ & não & $\operatorname{sim}$ & $\operatorname{sim}$ & $\operatorname{sim}$ \\
7 & $\operatorname{sim}$ & $\operatorname{sim}$ & $\operatorname{sim}$ & $\operatorname{sim}$ & $\operatorname{sim}$ & $\operatorname{sim}$ \\
8 & $\operatorname{sim}$ & $\operatorname{sim}$ & $\operatorname{sim}$ & não & não & $\operatorname{sim}$ \\
9 & $\operatorname{sim}$ & $\operatorname{sim}$ & $\operatorname{sim}$ & $\operatorname{sim}$ & $\operatorname{sim}$ & não \\
10 & $\operatorname{sim}$ & $\operatorname{sim}$ & $\operatorname{sim}$ & $\operatorname{sim}$ & $\operatorname{sim}$ & $\operatorname{sim}$ \\
\hline \hline
\end{tabular}

Tabela 3. Técnica condensação vertical de ondas contínuas (Grupo III) - preenchimento de canais laterais.

\begin{tabular}{ccccccc}
\hline \hline Dentes & $9 \mathrm{~mm}$ & $9 \mathrm{~mm}$ & $6 \mathrm{~mm}$ & $6 \mathrm{~mm}$ & $3 \mathrm{~mm}$ & $3 \mathrm{~mm}$ \\
\hline & Esquerda & Direita & Esquerda & Direita & Esquerda & Direita \\
1 & $\operatorname{sim}$ & $\operatorname{sim}$ & $\operatorname{sim}$ & $\operatorname{sim}$ & não & $\operatorname{sim}$ \\
2 & $\operatorname{sim}$ & $\operatorname{sim}$ & $\operatorname{sim}$ & $\operatorname{sim}$ & $\operatorname{sim}$ & $\operatorname{sim}$ \\
3 & $\operatorname{sim}$ & $\operatorname{sim}$ & $\operatorname{sim}$ & $\operatorname{sim}$ & $\operatorname{sim}$ & $\operatorname{sim}$ \\
4 & $\operatorname{sim}$ & $\operatorname{sim}$ & $\operatorname{sim}$ & $\operatorname{sim}$ & $\operatorname{sim}$ & não \\
5 & $\operatorname{sim}$ & $\operatorname{sim}$ & $\operatorname{sim}$ & $\operatorname{sim}$ & não & não \\
6 & $\operatorname{sim}$ & $\operatorname{sim}$ & $\operatorname{sim}$ & $\operatorname{sim}$ & $\operatorname{sim}$ & $\operatorname{sim}$ \\
7 & $\operatorname{sim}$ & $\operatorname{sim}$ & $\operatorname{sim}$ & $\operatorname{sim}$ & $\operatorname{sim}$ & $\operatorname{sim}$ \\
8 & $\operatorname{sim}$ & $\operatorname{sim}$ & $\operatorname{sim}$ & $\operatorname{sim}$ & $\operatorname{sim}$ & $\operatorname{sim}$ \\
9 & $\operatorname{sim}$ & $\operatorname{sim}$ & $\operatorname{sim}$ & $\operatorname{sim}$ & $\operatorname{sim}$ & $\operatorname{sim}$ \\
10 & $\operatorname{sim}$ & $\operatorname{sim}$ & $\operatorname{sim}$ & $\operatorname{sim}$ & $\operatorname{sim}$ & $\operatorname{sim}$ \\
\hline \hline
\end{tabular}

Tabela 4. Grupo I x /I x III - Comparação entre as mesmas variáveis entre grupos

\begin{tabular}{ll}
\hline \hline Distâncias do ápice radicular & Nível de Significância Estatística \\
\hline $3 \mathrm{~mm}$ à Grupo I X Grupo II & $\mathrm{p}=0.000^{*}$ \\
$3 \mathrm{~mm}$ à Grupo I X Grupo III & $\mathrm{p}=0.000^{*}$ \\
$3 \mathrm{~mm}$ à Grupo II X Grupo III & $\mathrm{p}=0.910$ \\
$6 \mathrm{~mm}$ à Grupo I X Grupo II & $\mathrm{p}=0.004^{*}$ \\
$6 \mathrm{~mm}$ à Grupo I X Grupo III & $\mathrm{p}=0.000^{*}$ \\
$6 \mathrm{~mm}$ à Grupo II X Grupo III & $\mathrm{p}=0.218$ \\
$9 \mathrm{~mm}$ à Grupo I X Grupo II & $\mathrm{p}=0.000^{*}$ \\
$9 \mathrm{~mm}$ à Grupo I X Grupo III & $\mathrm{p}=0.000^{*}$ \\
$9 \mathrm{~mm}$ à Grupo II X Grupo III & $\mathrm{p}=1.000$ \\
\hline
\end{tabular}

* Representa diferenças significativas estatisticamente $(\mathrm{p}<0.05)$

não houve diferença estatisticamente significante $(p>0.05)$.

A técnica de condensação lateral apresentou diferença estatisticamente significante $(\mathrm{p}<0.05)$ quando comparada à híbirda de Tagger e condensação vertical de ondas contínuas. Entretanto, não ocorreu diferença estatisticamente significante en- tre a técnica híbrida de Tagger e condensação vertical de ondas contínuas a 3, 6 e $9 \mathrm{~mm}$.

Seguem abaixo imagens dos espécimes e das radiografias digitais para visualização do preenchimento dos canais laterais, evidenciando-se o extravasamento do material obturador pelos canais laterais no 
Grupo II (híbrida de Tagger) e principalmente no Grupo III (técnica de condensação vertical de ondas contínuas). (Fig. 1 e 2 ).

\section{I SCUSSÃO}

A proposta do presente estudo foi avaliar radiograficamente a capacidade de obturação dos canais laterais artificiais utilizando as técnicas de condensação lateral, híbrida de Tagger e condensação vertical de ondas contínuas.

O meio de avaliação no presente estudo foi a radiografia digital. Esta foi escolhida por apresentar maior rapidez, "fidelidade" da imagem, segurança e facilidade de comunicação. Muitos autores concordam em que a imagem radiográfica é método de avaliação comparativa entre as técnicas de obturação, no qual se visualiza a distribuição do material obturador em toda a extensão do conduto ${ }^{16,17}$.

Optou-se por utilizar canais de dentes humanos extraídos ao invés de canais artificiais confeccionados em blocos de resina, por proporcionarrm uma maior proximidade com as variações anatômicas do
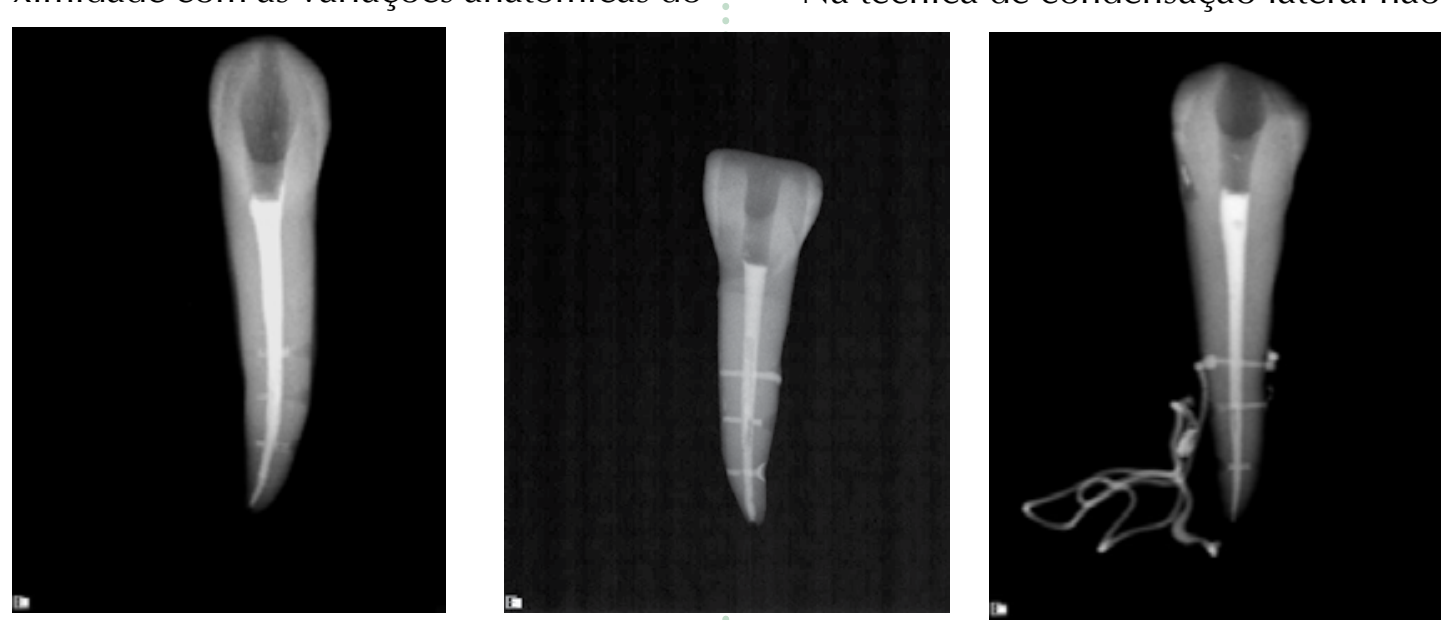

Figura 1. Imagens radiográficas dos dentes obturados: $A$ - condensação lateral; $B$ - híbrida de Tagger; C - condensação vertical de ondas contínuas.
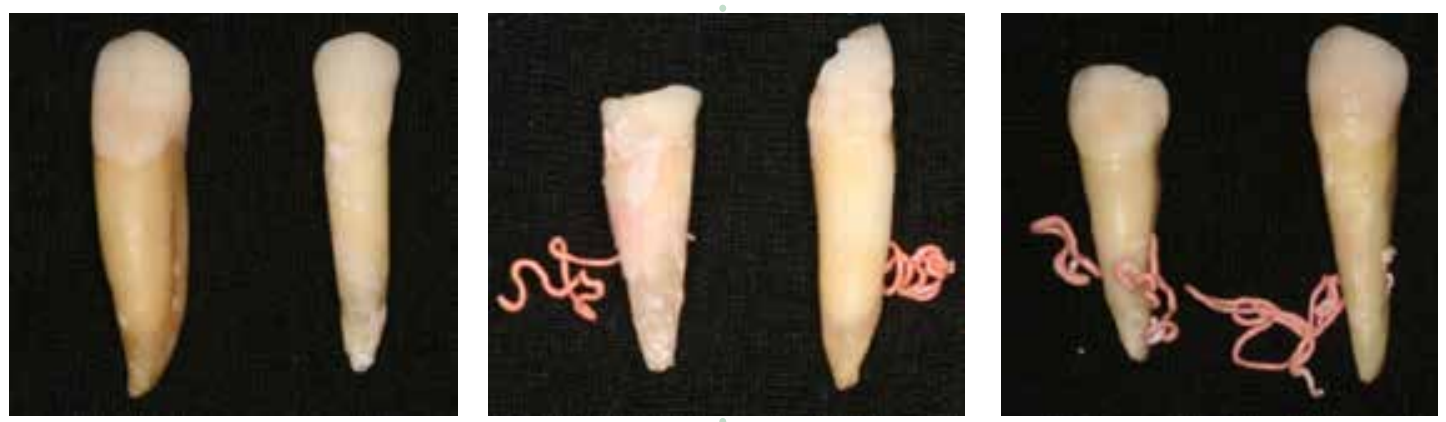

Figura 2. Imagens dos dentes obturados: $A$ - condensação lateral; $B$ - híbrida de Tagger; $C$ condensação vertical de ondas contínuas.
REBOUÇAS KS

PITHON MM

matos neto M

AVALIAÇÃO

RADIOGRÁFICA DO PREENCHIMENTO DE CANAIS LATERAIS

ARTIFICIAIS

UTILIZANDO TRÊS

TÉCNICAS DE

obturação

REV, ODONTOL.

UNIV, CID. São

paulo

$2013 ; 25(2): 126$ -

34, MAIO-AGO 
REBOUÇAS KS

PITHON MM

MATOS NETO M

$A \vee A L I A C ̧ \tilde{A} O$ RADIOGRÁFICA DO PREENCHIMENTO DE CANAIS LATERAIS ARTIFICIAIS UTILIZANDO TRÊS TÉCNICAS DE OBTURAÇÃO

\section{2}

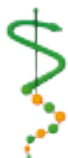

REV, ODONTOL.

UNIV, CID, SÃO

PAULO

2013; $25(2): 126$

34, MAIO-AGO

\section{ISSN 1983-5183}

se obtém uma massa obturadora homogênea, uma vez que o resultado final consiste em uma quantidade de cones de guta-percha pressionados uns contra os outros e unidos somente pelo cimento endodôntico. No entanto, já a técnica de condensação vertical de ondas contínuas apresenta maior homogeneidade, densidade e um preenchimento tridimensional do sistema de canais radiculares, sobretudo em sua porção apical, permitindo o selamento de canais acessórios, laterais e foraminas apicais com muita frequência ${ }^{14}$.

Dentre os resultados obtidos neste estudo, verificou-se que a técnica de condensação vertical de ondas contínuas apresentou a maior frequência de obturação dos canais laterais, tanto com cimento quanto com guta-percha, quando comparada à técnica híbrida de Tagger e à técnica de condensação lateral, sendo que esta última apresentou os canais simulados preenchidos apenas por cimento, estando de acordo com outros achados 2,13,20.

Porém, diante do estudo de Lima, Porto e Santos, em 200421, a técnica de condensação vertical de ondas contínuas (Schilder) apresentou capacidade de selamento compatível com as técnicas rotineiramente utilizadas, como a condensação lateral. Semelhante à pesquisa de Grenne, Wong e Ingram, em 1990, a qual não encontrou diferença significativa quanto ao selamento apical obturado pela condensação lateral ou técnica de condensação vertical de ondas contínuas.

Como resultado, a técnica de condensação lateral apresentou a maioria dos canais laterais sem obturar, semelhante aos achados de Brothman ${ }^{23}$ que, em 1981, comparou radiograficamente a técnica de condensação vertical com a técnica de condensação lateral quanto à obturação de canais laterais. A técnica de condensação vertical apresentou aproximadamente o dobro de canais laterais e acessórios obturados quando comparada com a técnica de condensação lateral. Com relação ao preenchimento do terço apical as técnicas apresentaram resultados semelhantes. Isso difere dos resultados obtidos na atual pesquisa, na qual o terço apical apresentou menor frequência de obturação nas duas técnicas.
Em um experimento com vinte caninos obturados, os quais passaram pelo processo de diafanização, foi evidenciado que a técnica híbrida de Tagger permitiu uma obturação mais homogênea com menos bolhas e falhas quando comparada à técnica da condensação lateral ${ }^{13}$, concordando com o presente estudo.

O trabalho realizado por Baish, Silveira e Martos ${ }^{9}$, em 2006, demonstrou que os canais simulados localizados nos terços cervical e médio apontaram diferença significativa em relação ao preenchimento quando obturados com as técnicas híbrida de Tagger e condensação lateral. Por outro lado, no terço apical essa diferença não foi evidenciada. Esses resultados discordam daqueles encontrados na atual pesquisa, pois esta demonstrou diferença significativa quando comparou a técnica híbrida de Tagger e a condensação lateral em todos os terços avaliados.

Semelhante ao presente estudo, a grande maioria das pesquisas que utilizaram a técnica híbrida de Tagger ressalta a sua efetividade quanto ao selamento e facilidade de execução. Entretanto, alguma controvérsia existe quanto ao nível em que se deve atuar o compactador em direção apical, a fim de manter-se a segurança da obturação quanto ao extravasamento e proporcionar um bom selamento do canal radicular ${ }^{6}$.

A falta do ligamento periodontal, cemento e osso alveolar proporcionou a saída do material obturador pelos canais laterais, diferentemente de in vivo, quando a presença desses elementos inviabilizaria esse extravasamento. Neste estudo, durante a realização das obturações pela técnica híbrida de Tagger e condensação vertical de ondas contínuas houve dificuldade no controle sobre a posição do material obturador em relação ao comprimento de trabalho ( $1 \mathrm{~mm}$ aquém do ápice) ocasionando, algumas vezes, o extravasamento desse material ${ }^{6,20}$.

Enquanto o presente trabalho avaliou as obturações através de confecção de canais laterais ${ }^{2,9,19,20,24}$, outras pesquisas comumente realizadas analisaram as obturações por meio de diafanização $\mathrm{O}^{7,8}$, corantes $^{4,5,6,7,16}$, cortes transversais ${ }^{6}$ ou microscópio ${ }^{4}$. Assim, ainda são poucas as 
publicações disponíveis que se propõem a realizar um estudo voltado especificamente para a confecção de canais laterais.

Com evidência de poucos estudos semelhantes, e por se tratar de um estudo in vitro, outras pesquisas devem ser realizadas a fim de conflitar e consolidar os resultados obtidos.

\section{CONCLUSÃO}

Com base na metodologia empregada e nos resultados obtidos é licito concluir que nenhuma das técnicas obturadoras avaliadas foi capaz de preencher todos os canais laterais simulados. Houve diferença significativa quando se comparou a técnica de condensação lateral com as outras técnicas, pois a mesma apresentou os piores resultados. Porém, quando se comparou a técnica híbrida de Tagger com a condensação vertical de ondas contínuas não ocorreu diferença significativa.

\section{REFERÊNCIAS}

1. FRACASSI, Larissa Dantas et al. Comparação radiográfica do preenchimento do canal radicular de dentes obturados por diferentes técnicas endodônticas. $R G O$, Porto Alegre, vol.58, n.2, abr./jun. 2010.

2. SILVA, Luciana Oliveira. Comparação entre a técnica de condensação lateral e a técnica termoplastificada na obturação endodôntica por meio de imagens radiográficas. 2009. $61 \mathrm{f}$. Trabalho de Conclusão de Curso (Especialização em Endodontia) - Centro Baiano de Estudos Odontológicos, Salvador.

3. LEONARDO, Mario Roberto; LEAL, Jayme Maurício. Obturação dos canais radiculares. In: LEONARDO, Mario Roberto; $L E A L$, Jayme Maurício. Endodontia: Tratamento dos canais radiculares. $4^{\circ}$ ed. São Paulo: Panamericana, 2005.

4. BEZERRA, Alexandre Gomes et al. Análise Comparativa do Selamento Apical promovido por diferentes Técnicas de Obturação em canais preparados com Instrumentação Automatizada. Ecler Endod, São Paulo, v.2, n.3, set. 2000.

5. VALERA, Marcia Carneiro; LEONARDO Mario Roberto; BONETTI, Idomeo. I. Cimentos endodônticos - selamento marginal apical imediato e após armazenamento de seis meses. Rev Odontol Univ, São Paulo, vol.12, n.4, oct./dec. 1998.
6. MORAES, Ivaldo Gomes et al. Técnica Híbrida de Tagger. RGO, Bauru, vol.48, n.3, p.141-4, jul./ago./set. 2000.

7. GRAF, Jacqueline Lucieri. Avaliação por meio de dentes transparentes do selamento apical promovido por três técnicas de obturação do sistema de canais radiculares. 2007. Dissertação (Mestrado em Odontologia). Área de concentração: Endodontia - Universidade do Grande Rio, Duque de Caxias.

8. MORGENTAL, Renata Dornelles; ZANATTA, Lílian Rosane Neuvald; RAHDEA, Nicole de Mello. Avaliação da obturação de canais laterais e secundários artificiais com diferentes técnicas obturadoras. Rev Odonto Ciênc, Porto Alegre, vol.23, n.3, p.273-7, dez./jan./fev./mar. 2008.

9. BAISH, Gisele Soriano; SILVEIRA, Luiz Fernando Machado; MARTOS, Josué. Análise radiográfica da repleção de canais secundários submetidos a duas técnicas de obturação. Rev Pós Grad, Pelotas, vol.13, n.2, p.139-44, 2006.

10. FERREIRA, Hamilton Luiz Junqueira; QUEIROZ DE PAULA, Marcos Vinícius; GUIMARÃES, Simone Maria Regone. Avaliação radiográfica de obturações de canais radiculares. Rev Odonto Ciênc, Porto Alegre, v.22, n.58, p. 340-5, out./dez. 2007.
REBOUÇAS KS PITHON MM matos neto M

AVALIAÇÃO RADIOGRÁFICA DO PREENCHIMENTO DE CANAIS LATERAIS ARTIFICIAIS UTILIZANDO TRÊS TÉCNICAS DE obTURAÇÃo

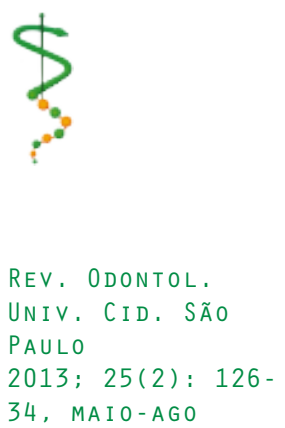


REBOUÇAS KS

PITHON MM :

MATOS NETO M

$A \cup A L I A C ̧ \tilde{A} O$ RADIOGRÁFICA DO PREENCHIMENTO DE CANAIS LATERAIS ARTIFICIAIS UTILIZANDO TRÊS TÉCNICAS DE OBTURAÇÃO
11. PINHEIRO, Bethânia Camargo; BRAMANTE, Alexandre Silva; HUSSNE, Renata Pardini. Influência da penetração de instrumentos, empregados na condensação lateral ativa, na qualidade da obturação de canais radiculares. J Appl Oral Sci, Bauru, vol.11, n.3, jul./set. 2003.

12. RAYMUNDO, Andressa et al. Análise radiográfica do preenchimento de canais laterais por quatro diferentes técnicas de obturação. RSBO, Curitiba, v.2, n.2, p.22-7, jun./jul. 2005.

13. CAMÕES, Izabel Coelho Gomes et al. Estudo Comparativo entre duas técnicas Obturadoras: Condensação Lateral X Híbrida de Tagger. Pesq Bras odontoped Clin Integr, João Pessoa, vol.7 n.3 p.217-22, set./dez., 2007.

14. SCHILDER, Hebert. Filling root canals in three dimensions. $J O E$, vol.32, n.4, p.281-90, 2006.

15. LOPES, Hélio Pereira; SIQUEIRA JR, Jose Freitas. Endodontia: Biologia $e$ Técnica. $3^{\mathrm{a}} \mathrm{Ed}$. Rio de Janeiro: Guanabara Koogan, 2010.

16. TARTAROTTI, Eder et al. Avaliação radiográfica da qualidade de obturações endodônticas. Rev Endod Pesq Ens, Cachoeira do Sul, vol.1, n.1, p.1-8, jan./Jun. 2005.

17. FERRAZ, Eduardo Gomes et al. Avaliação da qualidade de duas técnicas de obturação do canal radicular por meio de radiografia digitalizada. $R F O$, Salvador, v.14, n.2, p.126-31, maio/ago. 2009.
18. PERLICH, Malcolm; READER, Al; FOREMAN, Dennis. A scanning electron microscopic investigation of accessory on the pulpal floor of humans molars. J. Endod, vol.7, n.9, p.402-6, sept. 1981.

19. LIMONGI, Orlando et al. Comparação das técnicas de Condensação Lateral: Manual e com rotação Mecanizada. RGO, vol.52 n.1 p.42-4, 2004.

20. VILELA, Deyla Duarte et al. Evaluation of Interference of Calcium HydroxideBased Intracanal Medication in Filling Root Canal Systmes. J Contemp Dent Pract, vol.12, n.5, p.368-71, sept./oct. 2011.

21. LIMA, Mirella Emerenciano Massa; PORTO, Patricia de Oliveira Barbosa; SANTOS, Roberto Alves. Avaliação de três técnicas de Obturação Endodôntica. $R G O$, vol.52, n.1, p.13-8, jan./fev./ abr. 2004.

22. GREENE, Henry Anthony; WONG, Marston; INGRAM, Timothy. Comparação da Capacidade de Selamento de Quatro Técnicas de Obturação. J Endod, v.16, n.9, set. 1990.

23. BROTHAM, Peter. The comparative study of the vertical and lateral condensation of gutta-percha. J Endod, v.7, n.1, p. 27-30, jan. 1981.

24. PANTOJA, Carlos Augusto de Moraes Souto. Preenchimento e selamento marginal em canais laterais artificiais, após a utilização de diferentes técnicas obturadoras e cones de guta-percha. 2010. 71 f. Dissertação (Mestre em Clínica Odontológica). Área de concentração: Endodontia - Universidade Estadual de Campinas, Piracicaba.

Recebido em 21-01-2013

Aceito em 19-06-2013

\footnotetext{
REV. ODONTOL. UNIV. CID. SÃO PAULO
} $2013 ; 25(2): 126-$ 34, MAIO-AGO 